\title{
The Use of Capsule Endoscopy for the Investigation of Small Bowel Tumors: Experience from a United Kingdom Single Center
}

\author{
Reena Sidhu • Mark E. McAlindon
}

Published online: 13 July 2011

(C) Springer Science+Business Media, LLC 2011

We read with interest the article by Imaoka et al. [1] on the use of double-balloon enteroscopy (DBE) for the diagnosis of small bowel tumors. We would like to share our observations on using capsule endoscopy (CE) for the diagnosis of small bowel neoplasms. We conducted a study to evaluate the clinical presentation, endoscopic appearance, diagnostic and therapeutic impact of $\mathrm{CE}$ in the management of small bowel tumors. A retrospective review of consecutive patients that underwent $\mathrm{CE}$ with the findings of small bowel tumors over a 7-year period was conducted. Data was collected for patient demographics, indication of $\mathrm{CE}$, findings on $\mathrm{CE}$ including location of suspected tumor with subsequent follow-up data.

Twenty-four patients were found to have a small bowel tumor on CE. This represented $1.5 \%$ of all patients that underwent $\mathrm{CE}$ over the 7-year period. The indications included iron deficiency anemia $(n=9)$, overt bleeding $(n=11)$, and investigation of complications of celiac disease $(n=4)$. Patients had a mean number of 4.7 tests prior to $\mathrm{CE}$, which included gastroscopy, colonoscopy, small bowel radiology, computed tomographic scanning, mesenteric angiograms and red cell labeled scans. The mean age in the group was 55 years (range 34-83 years), which is similar to that reported by Trifan et al. [2] but lower than that reported by Imaoka et al. [1] in a Japanese population. The endoscopic appearances on CE included a mass or ulcerated mass lesion $(n=20)$ or isolated ulceration of mucosa with fresh bleeding $(n=3)$ and multiple ulcers with abnormal underlying mucosa $(n=1)$.

R. Sidhu $(\varangle) \cdot$ M. E. McAlindon

Department of Gastroenterology, Gastroenterology and Liver Unit, Royal Hallamshire Hospital, Sheffield Teaching Hospitals NHS Trust, Room P39, P Floor, Glossop Road, Sheffield S10 2JF, UK

e-mail: reena_sidhu@yahoo.com
The tumors were located in the jejunum in ten patients, in the ileum in 13 patients, and in the fourth part of the duodenum in one patient. The lesions were single in $96 \%$ of cases and multiple in $4 \%(n=1)$. Capsule retention occurred in four patients $(16.6 \%)$. Nineteen patients underwent surgery whilst 2 patients were treated with chemotherapy. The tumors were adenocarcinoma $(n=4)$, carcinoid $(n=2)$, gastrointestinal stromal tumor (GIST, $n=5$ ), lymphoma $(n=4)$, vascular tumors (hemangioma, angioma, angiomyolipoma, $n=6$ ), metastatic renal cancer $(n=1)$, glomus tumor $(n=1)$ and a benign fibroid tumor $(n=1)$. Three patients were treated palliatively.

CE like DBE is an important modality in the diagnostic work up of patients with small bowel tumors and it has a positive impact on patient management. The early use of $\mathrm{CE}$ in these patients should be encouraged as it often detects tumors missed by conventional modalities [3]. While CE is a non-invasive modality, it does not have the ability to obtain biopsies, hence both $\mathrm{CE}$ and DBE should be used complementary to each other for the diagnosis of small bowel tumors.

Conflict of interest No financial disclosures. No conflicts of interest.

\section{References}

1. Imaoka H, Higaki N, Kumagi T, Miyaike J, et al. Characteristics of small bowel tumors detected by double-balloon endoscopy. Dig Dis Sci. (Epub ahead of print). doi:10.1007/s10620-011-1741-8.

2. Trifan A, Singeap AM, Cojocariu C, Sfarti C, Stanciu C. Small bowel tumors in patients undergoing capsule endoscopy: a singlecenter experience. J Gastrointestin Liver Dis. 2010;19:21-25.

3. Rondonotti E, Pennazio M, Toth E, Menchen P, et al. Small-bowel neoplasms in patients undergoing video capsule endoscopy: a multicenter European study. Endoscopy. 2008;40:488-495. 